\title{
Injector Quantum Dot Molecule Infrared Photodetector: A Concept for Efficient Carrier Injection
}

\author{
Thomas Gebhard*
}

(Received 20 May 2011; accepted 25 July 2011; published online 13 August 2011.)

\begin{abstract}
Quantum dot infrared photodetectors are expected to be a competitive technology at high operation temperatures in the long and very long wavelength infrared spectral range. Despite the fact that they already achieved notable success, the performance suffers from the thermionic emission of electrons from the quantum dots at elevated temperatures resulting in a decreasing responsivity. In order to provide an efficient carrier injection at high temperatures, quantum dot infrared photodetectors can be separated into two parts: an injection part and a detection part, so that each part can be separately optimized. In order to integrate such functionality into a device, a new class of quantum dot infrared photodetectors using quantum dot molecules will be introduced. In addition to a general discussion simulation results suggest a possibility to realize such a device.
\end{abstract}

Keywords: Quantum dot infrared photodetector; Injection; Quantum dot molecule; High temperature operation; Responsivity

Citation: Thomas Gebhard, "Injector Quantum Dot Molecule Infrared Photodetector: A Concept for Efficient Carrier Injection", Nano-Micro Lett. 3 (2), 121-128 (2011). http://dx.doi.org/10.3786/nml.v3i2. p121-128

\section{Introduction}

The detection of infrared radiation $(3 \sim 30 \mu \mathrm{m})$ is of great interest for many applications such as astronomy, military, free space optical telecommunications, medicine and gas-detection. Since about four decades $\mathrm{HgCdTe}(\mathrm{MCT})$ photo-detectors are dominating the photo-detection in the long wavelength infrared (LWIR) spectral range. The main reason of this large success is the large quantum efficiency due to direct interband transition in a semiconductor bulk material with a long minority carrier lifetime, a high mobility and small effective mass of the electrons. But also the easy tunability of the detection wavelength by the change of the alloy composition within a lattice-matched material system makes MCT detectors perfectly suited for infrared detection. But the demand to fabricate large area focal plane arrays (FPA) pushed this technology to its limits, because an accurate control of the material composition is not trivial for large area devices. This is especially required for FPAs working in the LWIR and very long wavelength infrared (VLWIR) spectral range in order to achieve small values for the noise equivalent temperature difference (NEDT). As MCT detectors show a broad spectral response, the realization of multicolour infrared photodetectors is also not trivial due to spectral cross-talking effects $[1,2]$.

The onset of highly advanced epitaxial growth of III$\mathrm{V}$ materials opened a new chapter for the fabrication of infrared photodetectors. The possibility to grow of semiconductor optoelectronic devices within monolayer accuracy enables the tailoring of electronic properties beyond the restrictions given by nature as in the case of devices based on semiconductor bulk material. Quantum well infrared photodetectors (QWIPs) use intraband transitions to detect infrared radiation. These

Thomas Gebhard, Photonics Institute, Vienna University of Technology, Gusshausstr. 27-29/387, 1040, Vienna, Austria

*Corresponding author. E-mail: thomas.gebhard@tuwien.ac.at 
transitions can be tailored by the material combination of the heterostructure or by the variation of the quantum well $(\mathrm{QW})$ width. The major advantage of QWIPs is the relatively easy fabrication of homogeneous large area FPAs. The narrow-band response of QWIPs is also an advantage for multicolour applications because cross-talk between the colours can be inherently excluded, in contrast to multicolour MCTs. But nonetheless, MCTs show a better overall performance for operation temperatures above $60 \sim 70 \mathrm{~K}$, due to thermionic emission of the quantum-mechanically confined electrons within the quantum wells [2].

A further reduction of the systems dimensionality should in principle reduce the thermionic emission and in contrast to QWIPs absorption of normal incident light is inherently allowed [3]. The first intersubband quantum dot infrared photodetector (QDIP) was demonstrated nearly 15 years ago by Pan et al. [4]. Until today much research effort has been spent on QDIPs in order to achieve a device performance similar to QWIPs but at significantly higher temperatures. Notable success has been achieved in the meantime by several groups [5-11,28]. The most important approach to increase the detectivity, is the introduction of barriers in order to decrease the darkcurrent and/or to place the quantum dots (QDs) inside a quantum well (QW), these are the so called dot in a well (DWELL) structures [14]. The improvement of the detector performance in the case of the DWELL structure can be by part explained by an improvement of the refill mechanism of QDs with electrons, but also by an enhanced extraction efficiency of the photoexcited electrons within bound to bound transitions [9]. Bound to bound transitions are inherently more sensitive to normal incidence light compared to bound continuum transitions, so that the improvement of the photoexcited carrier extraction increases the quantum efficiency [15].

It is well known from experiment that the thermionic emission becomes a significant limiting factor especially in the LWIR and VLWIR-spectral range [7]. The result is that the responsivity decreases with increasing temperature, so that the detectivity is not only declining due to an increasing darkcurrent. Large values for the responsivity are also demanded in order to increase the frame rates in FPAs, so that a strategy must be found in order to preserve a large responsivity also for high operation temperatures [9]. In the following first part of the paper a QDIP-structure will be proposed where a quantum dot molecule (QDM) is used to optimize the electron capture and the spectral response separately. The final part of the paper presents a possible example for a device realization based on simulation results.

\section{Theory and general device structure}

In a first order approximation thermionic emission rate from a $\mathrm{QD}$ is proportional to $\exp \left[-\varepsilon_{\mathrm{QD}} / k_{B} T\right]$, where $\varepsilon_{\mathrm{QD}}$ denotes the ground state ionization energy, $k_{B}$ the Boltzmann constant and $T$ the temperature in Kelvin [12]. The electron capture by LO-phonon emission is on the other hand limited by the discrete spectrum of the QDs, i.e. the so called phonon bottleneck. As a result, very large values for the photoconductive gain are experimentally reported, i.e. in the range of $1 \sim 10^{6}$ [13]. The consequence of a low capture probability and an enhanced thermionic emission at elevated temperature results in a QD depopulation [16,39].

As already mentioned in the introduction the electron capture probability can by increased by placing the QDlayer within a $\mathrm{QW}$, i.e. a DWELL structure $[9,14,28]$. QW-width can be used to tune the final state of the transition and to achieve either bound to bound or bound to continuum transitions [15]. Especially at high temperatures an optimum QW-width must be found due to the trade-off between efficient electron capture and reemission, because with an increasing QW-width the activation energy of electrons in the QD groundstate (GS) becomes smaller, i.e. the onset of the $2 \mathrm{D}$ continuum shifts closer to the QD-GS [16], even if the QD-GS shifts to smaller energies with respect to the 3D-continuum of the bulk material [14]. The reemission of carriers from excited states (EXs) can decrease the resistance of the device at high temperatures $[13,39]$.

In order to optimize the electron capture independently of the spectral response and quantum efficiency of the detector, the QDIP structure can be split into two parts, an injection part and a detection part. In principle the structure consists of a pair of stacked QDs. Figure 1(a) depicts the conduction band profile of such a configuration for a cut through the QD-centre along the growth direction. The heights of the QDs are denoted with $h_{1}$ and $h_{2}$, measured from the bottom of the wetting-layer WL to the tip of each respective QD.

In the injection part of the structure (injectorDWELL, blue box) a QW with the width $\mathrm{d}_{1}$ (see Fig. 1(b) QW1) is placed below the QD-layer (QD1). This QW ensures that electrons are efficiently captured by the injector-DWELL. Barrier B covers the WL of the QD1-layer (WL1) and supports the capture of darkcurrent electrons [26].

The LWIR detection part of the structure is constituted by the detector-DWELL (as shown in Fig. 1, red-box). The two-dimensional (2D) system of the injection-DWELL, i.e. QW1 followed by WL1, is spatially separated from the detector part of the structure by a distance $\mathrm{D}$ in order to avoid carrier injection into the 2D-system of the detector-DWELL, i.e. the layers WL2 and QW2. An injection into QW2 would unnecessarily increase the darkcurrent, because thermal emission from 2D systems is very efficient due to the continuous density of states [16]. The close presence of a large 2D-carrier density can also enhance Auger-like 
scattering mechanisms [18] and this can additionally increase the emission of electrons in the detector-DWELL from the bound states to the continuum.

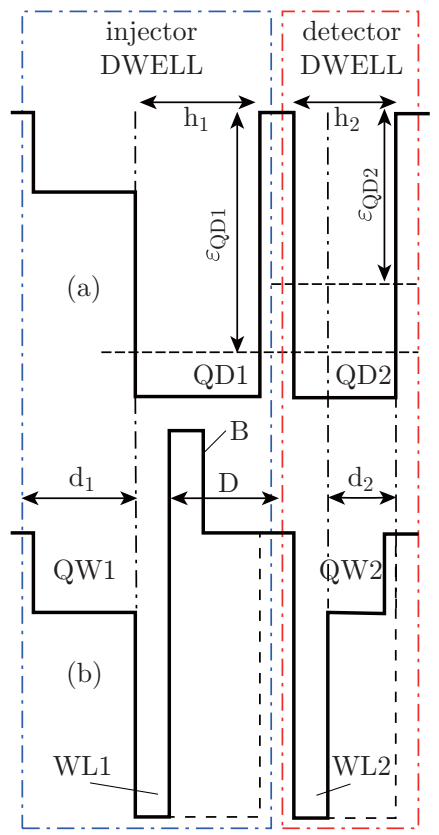

Fig. 1 In order to optimize carrier capture and the spectral response of the quantum dot infrared detector independently, the quantum dot infrared photodetector can be divided in an injector (blue box) and detector part (red box). Each part is realized as a dot in a well (DWELL)structure: (a) the top draft shows a cut through a couple of stacked quantum dots (QDs). In general the QDs have a different height $h_{1}$ and $h_{2}$. The activation energies $\varepsilon_{\mathrm{QD} 1}$ and $\varepsilon_{\mathrm{QD} 2}$ are defined as the energy difference between the QD-groundstate and the conduction band edge of the barrier-material, (b) the drawing on the bottom depicts a cut through the structure at a position without QDs. The quantum well QW1 with the corresponding width $\mathrm{d}_{1}$ ensures an efficient electron capture of the DWELL-injector. The barrier B covers the wetting layer WL1 of the quantum dot layer QD1 in order to increase the electron capture efficiency of the injector. QW1 is clearly separated from the DWELL-detector by a distance denoted with $\mathrm{D}$ in order to prevent an injection of electrons from the QW1 into QW2 with the width $\mathrm{d}_{2}$.

The activation energy $\varepsilon_{\mathrm{QD} 1}$ of the QD ground state of the DWELL-injector should be larger than that of the DWELL-detector $\varepsilon_{\mathrm{QD} 2}$ in order to suppress thermionic emission. The capture efficiency of the DWELL-injector can be optimized with an appropriate choice of the width $\mathrm{d}_{1}$ of QW1. The width $\mathrm{d}_{2}$ of QW2 should be as small as possible in order to avoid thermionic emission of confined carriers in QD2 and QW2 should only be used to maximize the spectral response of the detector $[9,15]$. Further, the spectral response of the detector-DWELL can be designed by the variation of the GS activation energy $\varepsilon_{\mathrm{QD} 2}$.
The key-functionality of the structure is that electrons are first captured by QW1. Some of the captured electrons further relax into the QD1-layer. Due to the proximity of the QD1- and QD2-layer in the order of $1 \sim 3 \mathrm{~nm}$, electrons can be injected well localized from the QD1- into the QD2-layer via resonant tunnelling or non-resonant tunnelling.

Resonant tunnelling is only possible in special cases where the energy levels between the electron tunnels perfectly coincide [34]. But in general, due to slight detunings, e.g. anti-crossing, resonant tunnelling can also be connected with acoustic phonon scattering [23,34]. Consequently, resonant tunnelling injection becomes more the exception than the rule for efficient electron injection into the detector-DWELL. Nonetheless resonant tunnelling is very fast, i.e. in the range of some tens of ps [24], when the involved states coincide energetically within a few $\mathrm{meV}$ [34].

Reischle et al. [24] found that non-resonant tunnelling rates increase exponentially with decreasing QD spacing and that tunnelling times are in the range of 500 ps for a barrier thickness of $3 \mathrm{~nm}$. For very small interdot layer spacing an offset of 425 ps was observed, most probably due to Pauli blocking. These values must be seen as an upper limit, because they have been evaluated at $4 \mathrm{~K}$ and multi-phonon processes become more important at higher temperatures [31].

The electron capture by the injector-DWELL must be fast compared with the electron tunnelling from QD1- to the QD2- layer in order to achieve large injection efficiency. Carrier capture times in conventional QDIPs can reach values in the range of $1 \mathrm{~ns}[13,38]$, i.e. QDIPs without QWs. As already mentioned in the outline the introduction of QWs in QDIP-devices can increase the carrier capture rate significantly [9]. The electron capture time for a DWELL-configuration is in the range of $100 \mathrm{ps}$ for a temperature of $200 \mathrm{~K}$ [28], i.e. it is shorter than the electron tunnelling time from the QD1- to the QD2-layer. QWELLs are also already successfully in use for injection interband QD-lasers with carrier relaxation times typically in the range of a few tens of ps [40]. In QDIPs this relaxation time is longer due to their unipolar nature and the smaller electron density in the devices, so that Auger-cooling does not happen [32] and the Auger-assisted tunnelling would be weak [18].

Another important design restriction for the proposed device is that the electron emission time from QD2 to the continuum should be smaller than the tunnelling time. This is necessary in order to ensure that the QD layer becomes permanently populated by electrons. The electron emission time at $200 \mathrm{~K}$ is in the range of some hundred ps [31], i.e. the same order of magnitude as the low temperature non-resonant tunnelling time mentioned before. This means that an efficient electron injection from the QD1- to the 
QD2-layer is principally possible, because the phononassisted inter-dot tunnelling rate increases with temperature. Small quantum dots have a larger capture time and as well as smaller emission efficiency [13]. This offers a possibility to balance the ratio between the QD1to QD2-layer tunnelling time and QD2 bound to continuum electron emission time.

Under unbiased conditions, the Fermi-level of the structure is determined by the doping of the injectorDWELL. Since the activation energy $\varepsilon_{\mathrm{QD} 1}$ of the injector-DWELL is larger as the activation energy $\varepsilon_{\mathrm{QD} 2}$ of the detector-DWELL, the thermionic emission becomes roughly reduced by the factor $\exp \left[-\left(\varepsilon_{\mathrm{QD} 1}-\right.\right.$ $\left.\left.\varepsilon_{\mathrm{QD} 2}\right) / k_{B} T\right]$ compared to the case of a conventional doped LWIR-DWELL detector.

Under biased conditions QW1 and B supply the QD1-layer with electrons in addition to the doping. As a consequence the Fermi-level increases as a function of bias at low temperatures. At temperatures above $130 \mathrm{~K}$ thermionic emission becomes significant due to multiphonon-absorption [31]. Without the capture of additional electrons, i.e. without applied bias voltage, the QD1-layer would empty at temperatures above $200 \mathrm{~K}$ [16].

The role of carrier capture by QW1 and the barrier B is to ensure a population of the injector-DWELL energy states at high temperatures, preferentially of the s- and p-states. Under such a situation the back-relaxation from the QD2- to QD1-layer becomes suppressed by Pauli-blocking [24].

It must also be noted that the density of states (DOS) below the steady state Fermi-level (if the Fermi-level is deeper than the GS of the detector-DWELL) is increased compared to the case of a conventional QDIPdevice, i.e. the injector-DWELL acts as an efficient electron reservoir. The optimum doping for QDIPs was found to be in the range of one to two electrons per QD [30]. The doping of QDIPs is in principle also limited by the small DOS of the QD-GS. The proposed device can in principle circumvent this limitation.

In principle the stacked QDs can be denoted as a quantum dot molecule (QDM) [18]. In order to simplify the discussion in the following, the proposed structure will be denoted as injection quantum dot molecule infrared photodetector (IQMIP).

\section{Device simulation}

In the following study a possible realization of an IQMIP-device will be presented in the $(\mathrm{In}, \mathrm{Ga}) \mathrm{As} /(\mathrm{Al}, \mathrm{Ga}) \mathrm{As} /(\mathrm{In}, \mathrm{Al}) \mathrm{As}$ material system. It is not the aim of the paper to analyze the electronic structure in detail, rather than to propose a general concept in order to improve the operation temperature of QDIPs. More information about QDMs can be found elsewhere [18-25].

The device simulation was performed with the free software NEXTNANO [29] by the use of the 8-band kp-method implemented in a 3D grid with a spatial resolution of $0.25 \mathrm{~nm}$. The material parameters have been taken from Vurgaftman et al. [17]. The QDs have been modelled by truncated pyramids with a lateral size of $18 \mathrm{~nm}$ and a height of $6 \mathrm{~nm}$.

The left part of Fig. 2 shows a detailed scheme of the structure, whereas on the right side the conduction band structure can be seen once for a cut through the wetting WL and once through the stacked QDs.

The barrier material consists of $\mathrm{Al}_{0.15} \mathrm{Ga}_{0.85}$ As. This material was chosen in order to improve the GaAs-QWs offer a stronger confinement. In the injector-DWELL a $5 \mathrm{~nm}$ thin GaAs-QW (QW1) is placed below the QD1layer. The WL of the QD1-layer is covered by a $1 \mathrm{~nm}$ thin $\mathrm{Al}_{0.42} \mathrm{Ga}_{0.58}$ As-layer [26].

The fabrication of asymmetric QDMs was already successfully demonstrated by Bracker et al. [35], where the "indium flush" method has been applied [36], i.e. the quantum dots are only partially capped after growth with a subsequent annealing step before they are then completely capped. This technique enables to engineer the height of the quantum dots. The energetic configuration of the QDM can be designed by the height of the QDs. But within the calculations it turned out that with this approach the energetic spacing between the two GS energies was limited when realistic quantum dot heights had been used. Therefore a modified approach has employed where the QDs are realized by different alloy compositions [33], i.e. the QD1-layer is realized by $\mathrm{Al}_{0.03} \mathrm{In}_{0.97} \mathrm{As}$ and the QD2layer by $\mathrm{In}_{0.5} \mathrm{Ga}_{0.5} \mathrm{As}$. The small migration rate of $\mathrm{Al}$ adatoms should force the formation of small QDs with a large density, increasing the quantum efficiency [27]. The reason not to use AlInAs QDs for the QD2- layer was that the composition of InGaAs-QDs is easier to control. QW2 is composed of GaAs and its thickness can be used in order optimize the quantum efficiency and the responsivity independently of the carrier injection [15].

Figure 3 shows the result of a simulation performed by the use of the 8-band kp-method. The straindistribution in the structure was taken into account by the minimization of the elastic energy. The influence of the strain on the conduction band as well as piezoelectric effects has been fully included.

Depending on the spatial dominance of the wavefunction the confined states can be assigned either to the injector- or the detector-DWELL. The energy difference between the two GSs $\varepsilon_{\mathrm{QD} 1}-\varepsilon_{\mathrm{QD} 2}$ is $73 \mathrm{meV}$, i.e. slightly larger than twice the LO-phonon energy of InAs-QDs [32], so that the application of a bias voltage can bring the GS of the injector-DWELL into a two-LO-phonon 


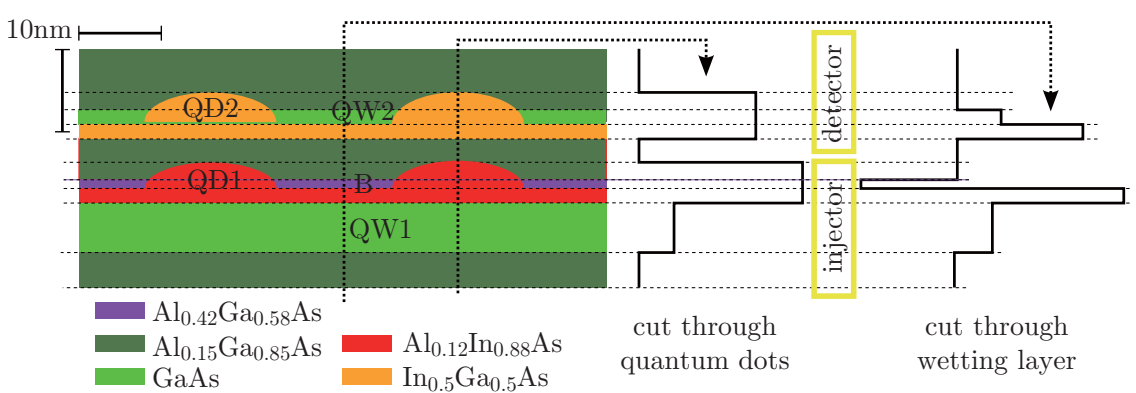

Fig. 2 The scheme shows the growth sheet of the proposed IQMIP-device (left). The right shows the bandstructure of the device once for a cut through the wetting layer and once through a couple of stacked quantum dots. Quantum dot layer 1 (QD1) and quantum well 1 (QW1) constitute the injector-dot in a well (DWELL) part of the IQMIP. QD2 and QW2 form the detector-DWELL part of the IQMIP with a spectral response in the long wavelength infrared range. At high temperatures the electron injection into the detector-DWELL occurs mainly by phonon assisted tunnelling through the QD1-layer.

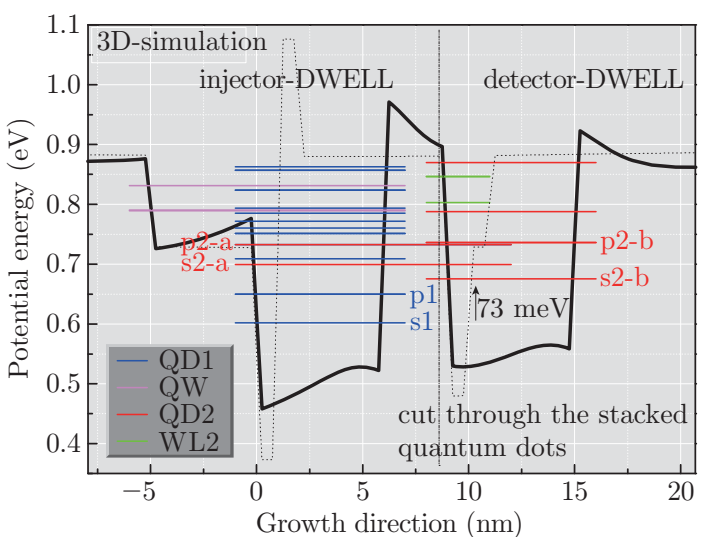

Fig. 3 The graph shows the result of a 3D simulation for the IQMIP-device shown in Fig. 2. The bound states of the system are marked by the set of horizontal lines and the spread of each line symbolizes the overlap of the corresponding wavefunction. The blue lines label the quantum dot (QD) states of the injector-DWELL (QD1), the violet lines label states that are strongly localized towards the quantum well (QW) of the injector-DWELL, the red lines label QD states of the detector-DWELL (QD2), and the green lines the states that are strongly localized along the wetting layer (WL). The s- and p-state of QD2 hybridize into bounding (s2-b, p2-b) and anti-bounding (s2-a, p2-a) states. The p2-b and $\mathrm{p} 2$-a are energetically spaced within the range of acoustical phonons. The energy difference between the injectorDWELL ground-state (GS) and the detector-DWELL-GS is $73 \mathrm{meV}$, i.e. approximately the energetic range of twice the LO-phonon energy. This enables to investigate resonant LO-phonon assisted injection as a function of the bias voltage. The bound to quasibound transition energy of the detector-DWELL is in the range of $120-170 \mathrm{meV}$, i.e. the transition energy between the GS and the WL-states.

resonance with that of the detector-DWELL. In a QDsystem the LO-phonon energy is not fixed at a single distinct value, i.e. different LO-phonon energies are possible: from the barriers, the WL, the QDs, and their respective facets. But also the broadening of phonons, due to strain and inhomogeneities can potentially enlarge the energetic window of two phonon assisted tun- nelling [32].

At temperatures above $130 \mathrm{~K}$ electron injection into the detector-DWELL should mainly occur also nonresonantly via EXs [24]. But a closer look to the results (see Fig. 3a) of the simulations offers an interesting feature of the structure: the p-state of QD2 hybridizes into bonding (p2-b) and anti-bonding states (p2-a) $[20,21,38]$. The corresponding energetic splitting of the states is only in the range of $2 \sim 3 \mathrm{meV}$, i.e. a carrier transfer between the QD1- and QD2-layer can occur by absorption of two to three acoustical phonons. The wavefunctions of the $\mathrm{p} 2-\mathrm{b}$ and $\mathrm{p} 2$-a states are shown in Fig. 4. It is interesting to see that the p2-a state is also significantly localized in the QD1-layer, but for the electron to become localized primarily in the QD2-layer a transition to the p2-b by phonon-scattering would be necessary. The probability of such a transition increases with temperature. In principle it does not make a large difference if the electrons are injected into the GS or the p-state, because once the electrons are injected into higher EXs of QD2 the electrons can further relax towards ground-state within a relatively short time-range of $30 \sim 60 \mathrm{ps}$ [41].

If an electric field is applied to the structure the electron injection from the QD1- to the QD2-layer becomes additionally supported and as mentioned before, if the electron capture of the injector-DWELL works, efficient Pauli blocking prevents the back-scattering of electrons from the detector- to the injector-DWELL.

The p-states of the injector are $26 \mathrm{meV}$ below the GS of the detector-DWELL, i.e. the density of states below the GS of the detector-DWELL is eight. This means that the electron-reservoir is significantly increased compared with the case of simple QDIP-devices.

The transition energy between the GS of the detector DWELL and the quasicontinuum, i.e. the WL-states (see Fig. 3a, green line) is around $120 \sim 170 \mathrm{meV}$. The thermal activation of photoexcited electrons from the $2 \mathrm{D}$-continuum of the WL to the continuum is very efficient at high temperatures $[16,30]$. 

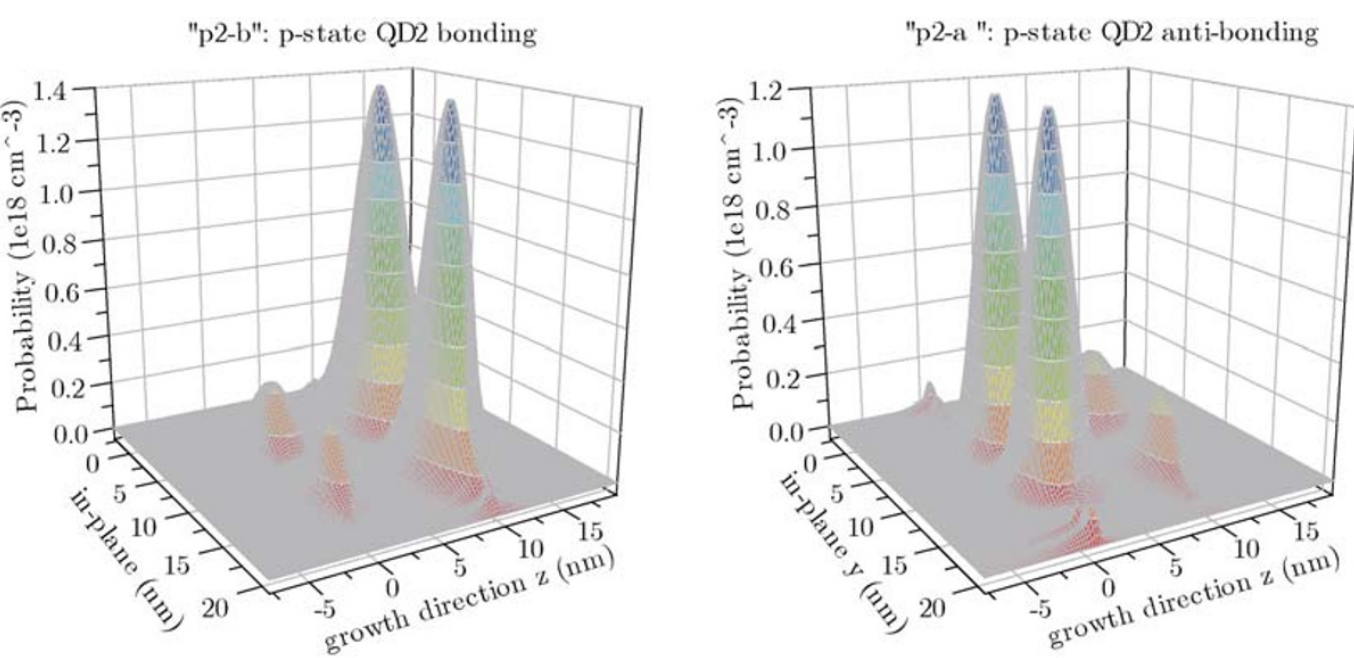

Fig. 4 The graph shows the wavefunctions of the bounding (left) and anti-bounding p-state (right) of the QD1 layer. The $\mathrm{z}$-axis shows the electron probability density along a $2 \mathrm{D}$ cut through the center of the QDs.

It is clear that the absorption spectrum of the injector-DWELL superimposes with the spectrum of the detector-DWELL. In order to realize multicolourdevices the spectrum must be optimized in order to minimize cross-talk effects. This can maybe be achieved with of the spectral dependence of the device on the bias voltage.

The inhomogeneous size distribution of the selfassembled QDs can be a limiting factor for some concepts presented within this paper. Different approaches are in use in order to decrease the size distribution of the QDs, e.g. the Indium flush method $[21,36]$. The use of this technique allows engineering the height of the QDs, but the size distribution of the lateral dimension stays unaffected. Consequently a different solution must be found in order to limit the influence of the lateral QD dimension variation. Stacking of QD is connected with a strong correlation via strain between the seeding QD-layer and the stacked QD layer [37]. This correlation is supposed to drive the size ratio between stacked QDs. Such a correlation reduces the influence of the inhomogeneous size distribution of self-assembled QD on the relative spacing between the GS of QD1 and QD2. In order to investigate this assumption, the lateral size of the both QDs was reduced from 18 to $15 \mathrm{~nm}$. The height was held constant due to the possibility to engineer it by the indium flush method. Figure 5 shows the result of this simulation. It can be seen that the relative shift of the s- and p-states of each QD with respect to each other is in the range of $1.2 \mathrm{meV}$, i.e. a two LO-phonon design is only weakly affected by the inhomogeneous size distribution of the QDs if the size ratio between QD1 and QD2 stays constant in the QDensemble. A more detailed study on this topic should bring more evidence if this approach would be realistic or not, but the first impression shows that the lateral size of stacked QD correlates [37].

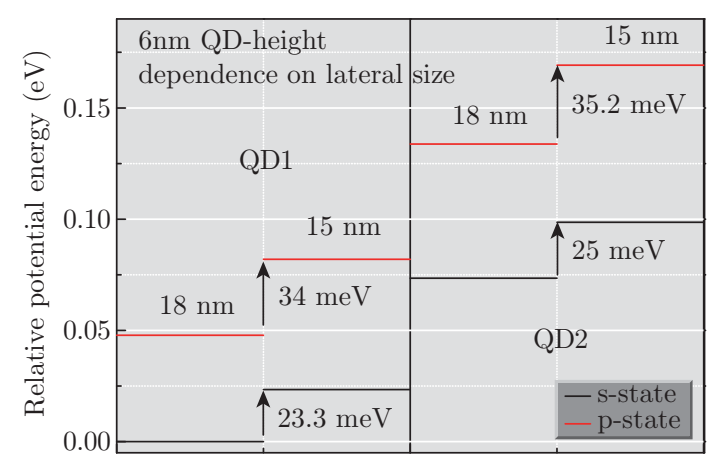

Fig. 5 Simulations with different lateral quantum dot (QD) dimension have been performed in order to investigate the influence of an inhomogeneous size distribution, i.e. for 18 and $15 \mathrm{~nm}$. The height of the QDs was kept at $6 \mathrm{~nm}$ for both simulation-runs, due to the possibility to employ the Indium-flush technique in order to achieve these dimensions. It can be seen that the energy difference between the s- and p-shell of the respective QDs is in the range of $1.2-1.7 \mathrm{meV}$.

\section{Conclusion}

In order to improve the operation temperature of a QDIP the device functionality can be separated in an injection and detection part. The electron injection can be realized by a QDM consisting of a double DWELL-structure. The GS of the injection DWELL is designed to be lower in energy than that of the detection-QWELL. This offers the possibility to inject carriers with the assistance of phonons into the detector-DWELL. The direct consequence of this design is that the static Fermi-level can be reduced in the active region compared to conventional doped LWIRQDIPs. An optimal design of the detector-QWELL should also minimize the reemission of electrons in order to maintain large values for the responsivity also at 
Nano-Micro Lett. 3 (2), 121-128 (2011)/ http://dx.doi.org/10.3786/nml.v3i2.p121-128

elevated temperatures.

As a proof of principle, a possible example to realize an IQMIP-device was presented in the InAlAs/InGaAs/GaAs material system. Different possible injection paths have been identified, i.e. resonant 2-LO phonon assisted injection between the GSs, acoustical phonon assisted tunnelling via the bonding and antibonding p-states of QD2, but also non-resonant tunnelling via EXs in general. Further, design restrictions have been roughly sketched, i.e. the electron capture time of the injector-DWELL should be faster than the tunnelling time between the QD1 and QD2-layer in order for efficient injection to occur. Additionally, the tunnelling time should be faster than the thermal emission time of electrons from the QD2-layer to the continuum. In principle, thermionic emission can be prevented by the use of small QDs, but also here a compromise must be found, because the reduction of the QD size reduces also the activation energy.

Optimal parameters and modification in the design must be found in a thorough experimental study, i.e. the non-resonant and resonant injection should be studied first as a function of temperature, and the energy difference between the GS of the injector- and the detector-DWELL.

\section{Acknowledgement}

The author thanks likes to thank Peter Vogl and his team at the Walter Schottky Institute in Munich for offering Nextnano in a free download. Further I like to thank Gernot Fasching to initialize the idea for this paper. Further I like to thank Govinda Lilley, Christoph Deutsch and Thomas Moldaschl from my institute to give valuable comments.

\section{References}

[1] A. Rogalski, Opto-Electron. Rev. 14, 84 (2006). http://dx.doi.org/10.2478/s11772-006-0012-2

[2] M. Z. Tidrow, W. A. Beck, W. W. Clark III, H. K. Pollehn, J. W. Little, J. K. Dhar, R. P. Leavitt, S. W. Kennerly, D. W. Beekman, A. C. Goldberg and W. R. Dyer, Opto-Electron. Rev. 7, 283 (1999). http://dx. doi.org/10.1063/1.2968128

[3] P. Martyniuk, S. Krishna and A. Rogalski, J. Appl. Phys. 104, 034314 (2008). http://dx.doi.org/10. 1063/1.2968128

[4] D. Pan, Y. P. Zeng, M. Y. Kong, J. Wu, Y. Q. Zhu, C. H. Zhang, J. M. Li and C. Y. Wang, Electron. Lett. 32, 1726 (1996). http://dx.doi.org/10.1063/1. 363742

[5] L. Jiang, S. S. Li, N. T. Yeh, J. I. Chyi, C. E. Ross and K. S. Jones, Appl. Phys. Lett. 82, 1986 (2003). http://dx.doi.org/10.1063/1.1540240
[6] H. Lim, S. Tsao, W. Zhang and M. Razeghi, Appl. Phys. Lett. 90, 131112 (2007). http://dx.doi.org/ 10.1063/1.2719160

[7] X. Lu, J. Vaillancourt and M. J. Meisner, Appl. Phys. Lett. 91, 051115 (2007). http://dx.doi.org/ 10.1063/1.2766655

[8] E. T. Kim, A. Madhukar, Z. Ye and J. C. Campbell, Appl. Phys. Lett. 84, 3277 (2004). http://dx.doi. org/10.1063/1.1719259

[9] S. D. Gunapala, S. V. Bandara, C. J. Hill, D. Z. Ting, J. K. Liu, S. B. Rafol, E. R. Blazejewski, J. M. Mumolo, S. A. Keo, S. Krishna, Y. C. Chang and C. A. Shott, IEEE J. quantum elect. 43 (2007). http://dx. doi.org/10.1109/JQE. 2006.889645

[10] S. Chakrabarti, A. D. Stiff-Roberts, X. H. Su, P. Bhattacharya, G. Ariyawansa and A. G. U. Perera, J. Phys. D: Appl. Phys. 38, 2135 (2005). http://dx.doi.org/ 10. 1088/0022-3727/38/13/009

[11] J. Shao, T. E. Vandervelde, A. Barve, W. Y. Jang, A. Stintz and S. Krishna, J. Vac. Sci. Technol. B 29, $03 \mathrm{C} 123$ (2011).

[12] V. Ryzhii, I. Khmyrova, V. Pipa, V. Mitin and M. Willander, Semicond. Sci. Technol. 16331 (2001). http:// dx.doi.org/10.1116/1.3562186

[13] H. Lim, B. Movaghar, S. Tsao, M. Taguchi, W. Zhang, A. A. Quivy and M. Razeghi, Phys. Rev. B 74, 205321 (2006). http://dx.doi.org/10.1116/1.3562186

[14] S. Krishna, J. Phys. D: Appl. Phys. 38, 2142 (2005). http://dx.doi.org/10.1088/0022-3727/38/13/010

[15] A. V. Barve, T. Rotter, Y. Sharma, S. J. Lee, S. K. Noh and S. Krishna, Appl. Phys. Lett. 97, 061105 (2010). http://dx.doi.org/10.1063/1.3475022

[16] F. Bras, P. Boucaud, S. Sauvage, G. Fishman and J. M. Gerard, Appl. Phys. Lett. 80, 4620 (2002). http:// dx.doi.org/10.1063/1.1487446

[17] I. Vurgaftman, J. R. Meyer and L. R. Ram-Mohan, J. Appl. Phys. 89, 5815 (2001). http://dx.doi.org/10. 1063/1.1368156

[18] P. Boucaud, K. S. Gill, J. B. Williams, M. S. Sherwin, W. V. Schoenfeld and P. M. Petroff, Appl. Phy. Lett. 77, 510 (2000). http://dx.doi.org/10.1063/1. 127027

[19] P. Boucaud, J. B. Williams, K. S. Gill, M. S. Sherwin, W. V. Schoenfeld and P. M. Petroff, Appl. Phy. Lett. 77, 4357 (2000). http://dx.doi.org/10.1063/ 1.1334912

[20] M. Korkusinski and P. Hawrylak, Phys. Rev. B 63, 195311 (2001). http://dx.doi.org/10.1103/ PhysRevB.63.195311

[21] A. Persano, A. Cola, A. Taurino, M. Catalano, M. Lomascolo, A. Convertino, G. Leo, L. Cerri, A. Vasanelli and L. Vasanelli, J. Appl. Phys. 102, 094314 (2007). http://dx.doi.org/10.1063/1.2812427

[22] M. F. Doty, J. I. Climente, M. Korkusinski, M. Scheibner, A. S. Bracker, P. Hawrylak and D. Gammon, PRL 102, 047401 (2009). http://dx.doi.org/ 10.1103/PhysRevLett. 102.047401 
[23] M. Scheibner, A. S. Bracker, D. Kim and D. Gammon, Solid State Commun. 149, 1427 (2009). http://dx. doi.org/10.1016/j.ssc.2009.04.039

[24] M. Reischle, G. J. Beirne, R. Roßbach, M. Jetter, H. Schweizer and P. Michler, Phys. Rev. B 76, 085338 (2007). http://dx.doi.org/10.1103/ PhysRevB. 76.085338

[25] C. Kammerer, S. Sauvage, G. Fishman, P. Boucaud, G. Patriarche and A. Lemaître, Appl. Phy. Lett. 87, 173113 (2005). http://dx.doi.org/10.1063/1. 2117621

[26] S. Y. Wang, S. D. Lin, H. W. Wu and C. P. Lee, Appl. Phy. Lett. 781023 (2001). http://dx.doi.org/ 10.1063/1.1347006

[27] X. H. Su, J. Yang, P. Bhattacharya, G. Ariyawansa and A. G. U. Perera, Appl. Phy. Lett. 89, 031117 (2006). http://dx.doi.org/10.1063/1.2233808

[28] H. S. Ling, S. Y. Wang, C. P. Lee and M. C. Lo, Infrared Phys. Techn. 52, 281 (2009). http://dx.doi. org/10.1016/j.infrared.2009.05.026

[29] Homepage Walter Schottky Institute, Munich.

[30] G. Jolley, L. Fu, H. H. Tan and C. Jagadish, Nanoscale 2, 1128 (2010). http://dx.doi.org/10. 1039/c0nr00128g

[31] M. De Giorgi, C. Lingk, G. von Plessen, J. Feldmann, S. De Rinaldis, A. Passaseo, M. De Vittorio and R. Cingolani, Appl. Phy. Lett. 79, 3968 (2001). http:// dx.doi.org/10.1063/1.1421235

[32] T. Mueller, F. F. Schrey, G. Strasser and K. Unterrainer, Appl. Phy. Lett. 83, 3572 (2003).http://dx. doi.org/10.1063/1.1622432

[33] A. R. Kovsh, A. E. Zhukova, A. Yu. Egorova, V. M. Ustinova, Yu. M. Shernyakova, M. V. Maximova, V.
V. Volovika, A. F. Tsatsul'nikova, Yu. V. Musikhina, N. N. Ledentsova, P. S. Kop'eva, D. Bimberg and Z. I. Alferov, J. Cryst. Growth 201, 1117 (1999). http:// dx.doi.org/10.1016/S0022-0248(98)01538-3

[34] K. Gawarecki, M. Pochwała, Anna Grodecka-Grad and P. Machnikowski, Phys. Rev. B 81, 245312 (2010). http://dx.doi.org/10.1103/PhysRevB.81. 245312

[35] A. S. Bracker, M. Scheibner, M. F. Doty, E. A. Stinaff, I. V. Ponomarev, J. C. Kim, L. J. Whitman, T. L. Reinecke and D. Gammon, Appl. Phy. Lett. 89, 233110 (2006). http://dx.doi.org/10.1063/1.2400397

[36] Z. R. Wasilewski, S. Fafard, J. P. McCaffrey, J. Cryst. Growth 201, 1131 (1999). http://dx.doi.org/10. 1016/S0022-0248(98)01539-5

[37] Q. Xie, A. Madhukar, P. Chen and N. P. Kobayashi, Phys Rev. Lett. 75, 2542 (1995). http://dx.doi.org/ 10.1103/PhysRevLett. 75. 2542

[38] B. Kochman, A. D. Stiff-Roberts, S. Chakrabarti, J. D. Phillips, S. Krishna, J. Singh and P. Bhattacharya, IEEE J. Quantum Elect. 39, 459 (2003). http://dx. doi.org/10.1109/JQE. 2002.808169

[39] L. Höglund, P. O. Holtz, H. Pettersson, C. Asplund, Q. Wang, S. Almqvist, S. Smuk, E. Petrini and J. Y. Andersson, Appl. Phy. Lett. 93, 103501 (2008). http:// dx.doi.org/10.1063/1.2977757

[40] P. Bhattacharya, J. Singh, H. Yoon, X. Zhang, A. Gutierrez-Aiken and Y. Lam, IEEE J. Quantum Elect. 32, 1620 (1996). http://dx.doi.org/10.1109/ 3.535367

[41] P. Aivaliotis, E. A. Zibik, L. R. Wilson, J. W. Cockburn, M. Hopkinson and N. Q. Vinh, Appl. Phys. Lett. 92, 023501 (2008). http://dx.doi.org/10.1063/1. 2833691 